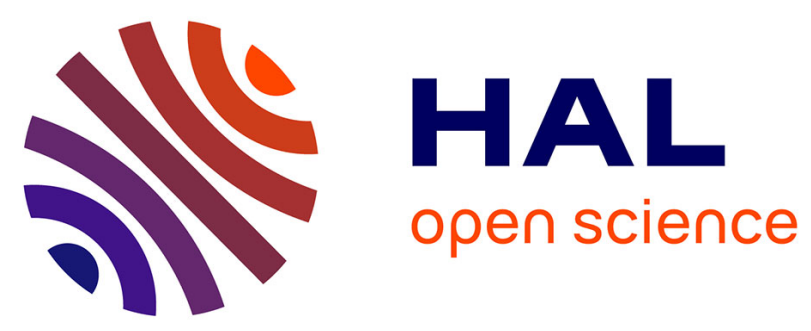

\title{
Modélisation électromagnétique des grands systèmes : l'apport des méthodes intégrales dans l'étude du foudroiement des avions
}

Ali Jazzar, Trung Son Nguyen, Edith Clavel, Jean-Michel Guichon, Olivier Chadebec, Gérard Meunier, Enrico Vialardi

\section{To cite this version:}

Ali Jazzar, Trung Son Nguyen, Edith Clavel, Jean-Michel Guichon, Olivier Chadebec, et al.. Modélisation électromagnétique des grands systèmes: l'apport des méthodes intégrales dans l'étude du foudroiement des avions. European Journal of Electrical Engineering, 2013, pp.65-86. 10.3166/ejee.16.65-86 . hal-03048119

\author{
HAL Id: hal-03048119 \\ https://hal.science/hal-03048119
}

Submitted on 9 Dec 2020

HAL is a multi-disciplinary open access archive for the deposit and dissemination of scientific research documents, whether they are published or not. The documents may come from teaching and research institutions in France or abroad, or from public or private research centers.
L'archive ouverte pluridisciplinaire HAL, est destinée au dépôt et à la diffusion de documents scientifiques de niveau recherche, publiés ou non, émanant des établissements d'enseignement et de recherche français ou étrangers, des laboratoires publics ou privés. 


\title{
Modélisation électromagnétique des grands systèmes
}

\section{L'apport des méthodes intégrales dans l'étude du foudroiement des avions ${ }^{1}$}

\author{
Ali Jazzar ${ }^{1}$, Trung-Son Nguyen ${ }^{1}$, Edith Clavel $^{1}$, \\ Jean-Michel Guichon ${ }^{1}$, Olivier Chadebec ${ }^{1}$, Gérard Meunier ${ }^{1}$, \\ Enrico Vialardi ${ }^{2}$
}

1. G2Elab, UMR5269

BP 46, F-38402 Saint Martin d'Hères cedex

Edith.Clavel@g2elab.grenoble-inp.fr

2. CEDRAT

15, Chemin de Malacher - Inovallée, F-38246 Meylan Cedex

Enrico.Vialardi@cedrat.com

RÉSUMÉ. Dans une vision de transports plus économiques, l'apport des matériaux composites est une solution technologique importante pour l'industrie aéronautique, même si cela ouvre de nouvelles questions de compatibilité électromagnétique sur l'ensemble du système et nécessite l'utilisation d'outils de simulation adaptés. La méthode intégrale PEEC (Partial Element Equivalent Circuit) est une approche pertinente pour la simulation électromagnétique de ces grands systèmes où beaucoup d'espace vide existe entre les conducteurs. Néanmoins, les codes PEEC basses fréquences actuellement disponibles montrent des limites dans le traitement des objets de grande taille qui sont à modéliser pour étudier la problématique du foudroiement des avions. Cet article illustre les récents développements effectués afin d'augmenter les possibilités de simulations électromagnétiques offertes par le logiciel InCa3D et discute des résultats de simulation et de mesures sur un caisson en matériau composite, échantillon représentatif d'un avion.

ABSTRACT. For economic reasons, using composite material remains a solution for aeronautic industries. But their efficiency concerning the electromagnetic compatibility of systems require some studies. For that the use of adapted simulations tools is unavoidable. The integral method PEEC (Partial Element Equivalent Circuit) is well adapted to model large systems where a lot of space is air around the conductors. Nevertheless, the actual low frequencies PEEC tools are limited for the description of large systems which is the case of airplanes to study the lightning effect. This article describes the abilities of InCa3D tool to 
simulate electromagnetic effects. The modeling process is detailed and results are discussed and compared to measurements on a representative sample of airplane configuration. The study proposes also some improvements of the tool concerning the solving step. They consist in using the Fast Multipole Method associated to an iterative solving. Their performance is compared on the same sample with the actual tool InCa3D. The obtained results allow then to plan the modeling of a whole system.

MOTS-CLÉS : foudre, simulation électromagnétique fréquentielle et temporelle, méthode PEEC. KEYWORDS: lightning, electromagnetic modeling, frequency and time analysis, PEEC method.

\section{Extended Abstract}

For economic reasons, using composite material remains a solution for aeronautic industries (less heavy, very robust, less fuel). But their efficiency regarding the electromagnetic compatibility performance requires some studies. Indeed since its physical properties like resistivity are quite different from that of aluminum, the Faraday effect of the skin of the plane does not remain as efficient in case of lightning. So it is necessary to be able to evaluate the possible direct and indirect effects of lightning on the electrical systems inside the aircraft. One of the more representative data is the current flowing in cables after a lightning on the skin. To predict this current, the use of adapted simulations tools is unavoidable. The sizes of the structure under study can be very big so that commonly used methods such as Finite Element Method (FEM) or Finite Difference Method (FDTD) are not adapted. The integral method PEEC (Partial Element Equivalent Circuit) is well adapted to model large systems where a lot of space is air around the conductors. Nevertheless, the actual low frequencies PEEC tools are limited for the description of large and complex systems. The number of elements and the use of full matrices limit their possibilities.

In this article, some improvements of the modeling process concerning the solving step are proposed. They consist in using the Fast Multipole Method associated to an iterative solving and to improve the algorithm for circuit equations research.

They have been included in $\mathrm{InCa} 3 \mathrm{D} \otimes$ tool which is based on PEEC method to simulate electromagnetic effects. The performance, abilities and modeling process are detailed and results are discussed and compared to measurements on a representative sample of airplane configuration. The good agreement between simulations and measurements proves that this modeling process is a good help for designer to develop efficient protecting devices. Harmonic and time evaluations have been carried out. For the first one, only PEEC method is used through InCa3D soft. And for the second one, InCa3D is able to automatically generate an electrical equivalent circuit for a circuit solver like SPICE, Saber, etc. For the studied case, 
Portunus ${ }^{\circledR}$ has been used. The obtained results allow then to plan the modeling of a whole system.

\section{Introduction}

L'industrie aéronautique conçoit les avions de demain en suivant deux tendances : plus électrique et plus composite. Si les intérêts sont multiples et connus, certaines conséquences sont moins bien maîtrisées. Entre autres, la vulnérabilité des réseaux électriques exposés aux effets indirects de la foudre demande la conception de protections innovantes. Cela suppose une meilleure évaluation des courants induits dans la structure devenue complexe de par les différentes échelles qui la caractérisent, mais également de par l'introduction de matériaux de conductivités très différentes (rapport de l'ordre de 8000 entre l'aluminium et le composite). En outre, la nécessaire prise en compte de ces contraintes en phase amont d'un projet ainsi que l'exigence de diminution de coût fait que l'utilisation de moyens numériques de simulation est privilégiée.

Dans ce contexte, il apparaît que certaines méthodes sont préférables pour la modélisation des effets indirects de la foudre. La nature de cette source d'excitation guide le choix de la méthode. En effet, la forme d'onde du courant foudre appliquée dans un calcul 3D est normalisée, de type bi-exponentiel. C'est donc un signal impulsionnel et plutôt basses fréquences (de l'énergie jusqu'à $1 \mathrm{MHz}$ environ) qui attaque l'avion ou un de ses sous-systèmes. À l'intérieur de ceux-ci, les lignes de courant se répartiront entre les différentes parties qui les composent au prorata de leurs résistances (composantes basses fréquences) et inductances (composantes moyennes fréquences). Ainsi disposer d'un modèle électrique équivalent des dispositifs d'étude permettra d'évaluer l'impédance et de prédire le courant dans chaque partie du dispositif. La méthode PEEC basses fréquences peut donc répondre à ces besoins puisqu'elle permet efficacement (c'est-à-dire sans mailler l'air) de faire une projection d'un modèle 3D de la structure vers un modèle de type circuit (éléments R, L, M) (Roudet et al., 2004). Le caractère basses fréquences du signal agresseur permet de s'affranchir du calcul des capacités parasites. Ainsi un schéma électrique équivalent à constantes localisées peut être évalué et utilisé dans une simulation temporelle. En outre, à des fins d'amélioration de la structure vis-à-vis d'éventuelles conséquences d'une agression foudre, il est important de pouvoir décrire le problème en utilisant des paramètres géométriques ou physiques (position des faisceaux de câbles, nombre et position des rivets par exemple), potentialité disponible naturellement grâce à la méthode PEEC.

Néanmoins, les codes PEEC basses fréquences actuellement disponibles montrent des limites dans le traitement de la taille des objets qui sont à étudier pour la problématique foudre sur l'avion. En effet, la méthode PEEC (et d'une façon générale toutes les méthodes intégrales) conduit à la résolution de systèmes linéaires pleins. Par conséquent si $\mathrm{N}$ est le nombre d'inconnues d'un problème, la place mémoire nécessaire au calcul évolue en $\mathrm{N}^{2}$ et la durée de résolution en $\mathrm{N}^{3}$ si des 
algorithmes directs classiques sont utilisés. Le logiciel InCa3D ${ }^{\circledR}$ s'appuie sur la méthode PEEC et est co-développé par G2Elab et CEDRAT. Ses limites actuelles sont directement en lien avec celles pointées ci-dessus.

Traditionnellement, dans le domaine de l'aéronautique, ce sont les méthodes FDTD (Tirkas et al., 1993) qui sont privilégiées pour évaluer les répartitions des courants. Néanmoins leur utilisation dans le cadre de la problématique foudre est limitée du fait de la grosseur des modèles (nombre d'inconnues élevé et augmenté par la présence du matériau composite) et du pas de temps nécessaire pour le type de signal concerné (Gutiérrez et al., 2011). Aussi dans la perspective de fournir une réponse industrielle à cette problématique, une collaboration née dans le cadre du projet PREFACE a permis d'évaluer et de comparer les différentes méthodes disponibles qu'elles soient classiquement utilisées pour traiter les interactions foudre comme la FDTD (Perrin et al., 2012) ou qu'elles s'appuient sur des méthodes intégrales. Par la suite, une fois la méthodologie de modélisation établie, des campagnes de mesures destinées à valider les modèles ont permis de conclure sur la validité des choix adoptés selon que l'on se situe dans une phase de préconception ou de validation de solutions.

Cet article expose les améliorations qui ont été apportées au solveur PEEC inductif à travers la modélisation d'une partie représentative d'un avion. Dans un premier temps, une description de la maquette du caisson d'avion est présentée. La partie 3 de l'article se propose de détailler les améliorations qui permettront ainsi de traiter les systèmes de grandes dimensions. Une fois le processus de modélisation amélioré, la partie 4 de l'article compare les performances de résolution entre la version actuelle du logiciel InCa3D et celle développée à la fois en termes de rapidité, taille des matrices résolues mais aussi pertinence des résultats sur le prototype étudié. Quelques pistes de discussion sur la modélisation électromagnétique sont abordées à travers les répartitions de courant sur le caisson en fonction de la fréquence et des résultats de simulation temporelle à l'aide du logiciel Portunus ${ }^{\circledR}$ distribué par Cedrat. La dernière partie de l'article permet de conclure et de proposer des suites à cette étude.

\section{Cas d'étude}

L'objet d'étude est un échantillon représentatif du caisson de grande taille correspondant à la carlingue d'un avion composite homogène (figure 1 à gauche). Il est constitué de plaques en matériau composite liées entre elles par des équerres isolantes et maintenues par des rivets. Le projet dans le cadre duquel a été conçu ce caisson n'étant pas terminé, les données à la fois géométriques et physiques de cette structure test ne sont pas disponibles.

Dans un premier temps, un gros travail à la fois de description fidèle ainsi que de simplifications a été effectué afin de pouvoir mener à bien une modélisation dans des temps de simulations raisonnables. La principale simplification a consisté à 
décrire le sandwich "équerre en aluminium+plaque de composite+rivets » par un matériau équivalent dont les propriétés physiques ont été définies à l'aide de mesures exhaustives. A l'intérieur du caisson, des câbles métalliques courent le long des différentes parois. Leur mode de connexion au reste du dispositif a été décrit de façon précise afin de prendre en considération toutes les caractéristiques électriques parasites. Le dispositif décrit est présenté figure 1 à droite.

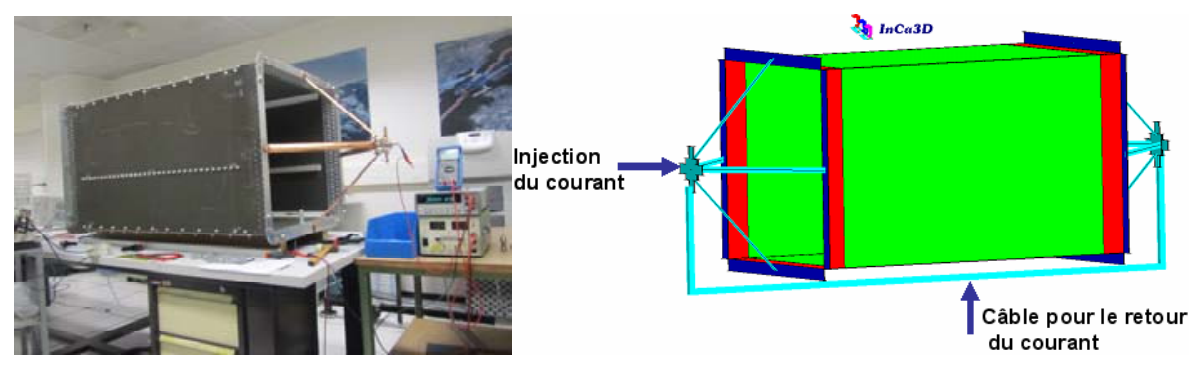

Figure 1. Caisson : dispositif réel (à gauche) et dispositif modélisé dans InCa3D (à droite)

Le principal impact de l'introduction du matériau composite pour la carlingue intervient dans la perte du rôle de cage de Faraday qu'assurait la peau tout en aluminium et qui permettait ainsi de protéger les structures internes en offrant au courant foudre un chemin privilégié d'écoulement. Pour analyser la répartition du courant foudre entre les câbles métalliques et la peau carbone, un courant est injecté ponctuellement sur le caisson. Ainsi, l'étude des comportements des courants sur les surfaces composites de la boîte, et des courants de court-circuit dans les fils électriques placés à l'intérieur de la cavité peut être entreprise. En effet, il apparaît des phénomènes complexes de redistribution des courants dans l'ensemble de la structure liés aux effets de peau et de proximité, du fait de la géométrie complexe, des caractéristiques physiques des éléments et du contenu harmonique des signaux impliqués. L'étude de ces nombreux couplages est permise dès lors qu'est disponible un outil de simulation efficace, décrit dans la prochaine section. Cette étude est abordée à la fois de manière numérique et expérimentale avec la volonté de comprendre les effets de la foudre sur ces matériaux composites et pour évaluer la méthode PEEC et la comparer aux méthodes classiques FDTD. Une campagne de mesures, en fréquentiel et en temporel, est effectuée en collaboration avec l'entreprise EADS et le laboratoire ONERA au cours de laquelle plusieurs configurations sont imaginées. 


\section{Méthode PEEC et développements.}

\subsection{Présentation du logiciel}

Le logiciel InCa3D $($ Inductance Calculation), codéveloppé par le G2Elab et la société Cedrat, est un logiciel de prédétermination des éléments parasites de couplage au sein des structures complexes d'électrotechnique et d'électronique de puissance. Il s'appuie sur la méthode PEEC et permet d'une part, de calculer efficacement les densités de courant dans les connexions électriques en régime de basses fréquences et forts courants, et d'autre part, d'extraire, pour chaque fréquence des caractéristiques électriques $(\mathrm{R}, \mathrm{L}, \mathrm{M}$ résistance, inductances propres et mutuelles) de conducteurs, de forme plus ou moins complexe, placés dans l'air. La méthode est détaillée par Roudet et al. (2004) et un exemple d'application peut être trouvé dans Clavel et al. (2011). Ainsi, le synoptique du principe de simulation sous InCa3D qui apparaît sur la figure 2 peut être découpé en cinq étapes.

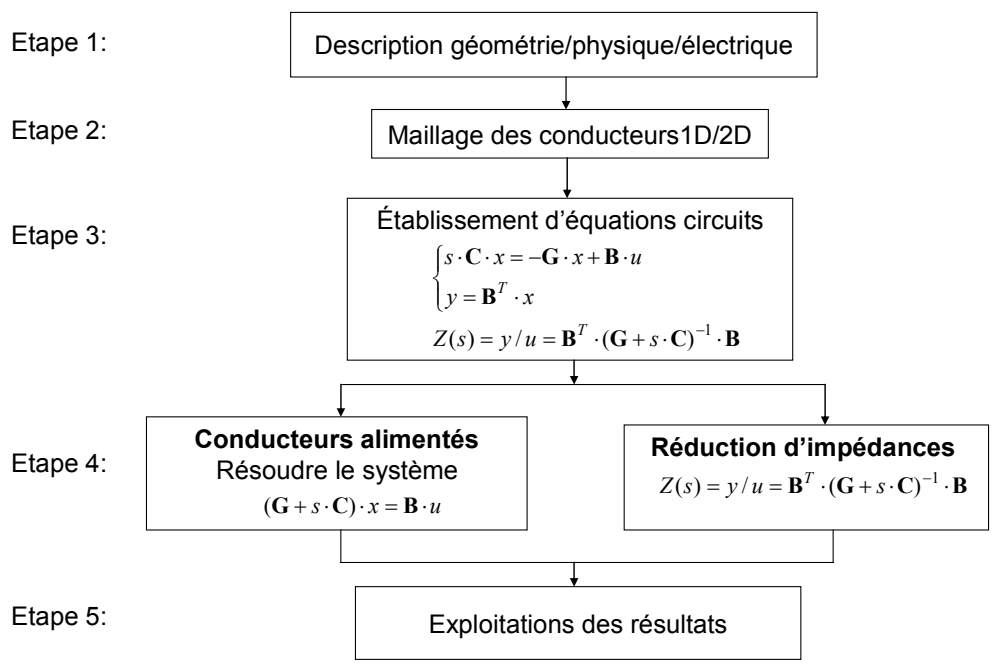

Figure 2. Synoptique du principe de modélisation du logiciel InCa3D

Étape 1. Description du problème à modéliser sur InCa3D : l'ensemble des conducteurs physiques ainsi que le circuit électrique (sources de courant ou de tension et éléments passifs de type résistance, inductance, ou condensateur et liaison équipotentielles entre les conducteurs).

Étape 2. Subdivision des conducteurs en plusieurs éléments dans lesquels la densité de courant est supposée uniforme. La technique de maillage est adaptée à la forme des conducteurs ainsi qu'à la fréquence donnée en 1. 
Étape 3. Calcul des éléments $\mathrm{R}$, $\mathrm{L}$ et $\mathrm{M}$ de chacune des subdivisions physiques et écriture des équations de relation courant/tension sur les éléments maillés et sur le reste du circuit électrique. Les relations circuit électrique sont utilisées pour décrire le système sous forme mathématique :

$$
\left\{\begin{array}{c}
s \mathbf{C} x=-\mathbf{G} x+\mathbf{B} u \\
y=\mathbf{B}^{T} x
\end{array}\right.
$$

où :

- $\mathbf{x}$ : vecteur des inconnues du système à résoudre (des courants de mailles indépendantes et des tensions de mailles indépendantes voir 3.2 pour la justification de ce choix).

- C : matrice description des éléments inductifs et capacitifs (éléments propres sur la diagonale et mutuels ailleurs),

- $\mathbf{G}$ : matrice description des éléments résistifs

- B : matrice incidence d'entrée/sortie du système

- u : les entrées (sources de courant/tensions)

$-\mathbf{y}$ : les sorties, complémentaires électriques de $\mathbf{u}$

$-\mathrm{s}=2 . \pi$.f (avec $\mathrm{f}$, la fréquence du problème)

Étape 4. Deux possibilités :

- Avec l'application Conducteurs alimentés, l'utilisateur analyse la structure dans son environnement électrique, le résultat principal est le courant qui circule dans les conducteurs.

- Avec l'application Réductions d'impédances, l'utilisateur déduit du calcul PEEC les caractéristiques équivalentes qui l'intéressent. Le résultat principal est l'impédance de portions du circuit définies par l'utilisateur à l'aide de sondes d'impédance.

Étape 5. Exploitations des résultats :

- Conducteurs alimentés : à partir du courant, un certain nombre de grandeurs électromagnétiques peuvent être calculées :

- l'induction magnétique B au voisinage des conducteurs,

- l'effort électrodynamique entre conducteurs (force de Laplace),

- la puissance dissipée dans les conducteurs (pertes par effet Joule).

- Réductions d'impédances: les impédances des conducteurs, quant à elles, peuvent être injectées dans un simulateur de circuit de type PORTUNUS, SPICE, SABER, ou exportées dans les langages de type MODELICA, VHDL-AMS, etc. 


\subsection{Limitations et développements nécessaires}

Lorsque les systèmes à résoudre sont de tailles importantes avec beaucoup d'air autour du dispositif, comme c'est le cas des réseaux de bord d'avions à l'étude, les méthodes de type FTDT conduisent à un nombre d'éléments de maillage trop élevé pour permettre une résolution dans des temps raisonnables. Mais avec la méthode PEEC inductive comme présentée précédemment, cela devient aussi critique. Cette méthode semble moins adaptée en l'état et nécessite des développements. Des travaux sur cette méthode intégrale dans le domaine temporel confirment son potentiel indéniable (Ruehli et al,. 2003). Les travaux présentés dans cet article se concentrent sur de récentes améliorations qui ont permis de contourner les difficultés précédemment mentionnées. Leur mise en œuvre intervient surtout au niveau des étapes 3 et 4 .

Dans l'étape 3, abordée en partie 3.2.1, le travail consiste à accélérer la recherche de mailles indépendantes pour faciliter les phases de résolutions de systèmes linéaires.

Dans l'étape 4, lors de la résolution du système, nous voulons résoudre les équations de circuit issues de l'étape précédente de façon plus efficace que dans la version actuelle. Une combinaison de deux algorithmes est donc présentée en section 3.2.2, l'un FMM (Fast Multipole Method) permet de compresser la matrice de description du système. Le recours à la FMM oblige à utiliser un solveur itératif (GMRes) pour résoudre le système. Une description de son principe est proposée, ainsi que les questions de préconditionnement qui l'accompagne.

D'un point de vue quantitatif, le principal objectif visé par ces développements est de gagner un ordre de grandeur dans la taille du système à résoudre et de permettre une résolution sur un «ordinateur banal». On peut enfin noter que si l'exposé n'aborde que la description des méthodes et des algorithmes, les développements effectués sont codés en langage java (avec mise en place d'une parallélisation afin d'exploiter le calcul multiprocesseur).

\subsubsection{Développement pour la mise en place des équations de circuit}

Afin de déterminer les courants et tensions d'un circuit électrique, deux approches sont possibles : l'approche par mailles indépendantes et l'approche par nœuds indépendants.

L'approche par nœuds indépendants, connue sous le nom d'analyse nodale est inadaptée pour les circuits avec de nombreuses inductances mutuelles. En effet, elle aboutit à l'écriture d'un système généralement mal conditionné (Kamon et Philips, 1994). Par contre, l'analyse des mailles indépendantes est plus efficace pour ce type de topologie de circuit puisqu'elle réduit au maximum le nombre d'équations de circuit tout en garantissant un meilleur conditionnement du système d'équations résultant. 
Malgré ce net avantage, l'algorithme proposé actuellement dans InCa3D est très gourmand en place mémoire et en temps de calcul lorsqu'il est appliqué sur des structures 3D complexes. En effet, il est basé sur une méthode algébrique de détection générale pour trouver les mailles indépendantes. Elle est appliquée de manière naïve dans le sens où elle ne tient pas compte de la topologie des structures.

En effet, pour l'identification des mailles indépendantes, le maillage PEEC est transformé en un graphe contenant les nœuds et les branches électriques sans tenir compte des mutuelles entre elles. L'application de la méthode de détection de maille indépendante est basée sur ce graphe. Un exemple est détaillé figure 3. À gauche, la méthode algébrique de détection générale est employée pour identifier totalement les mailles indépendantes du système et 21 sont les mailles trouvées.

Or, à l'image de l'exemple donné figure 3, typique d'un maillage PEEC, on constate qu'il est possible de décomposer le système à l'aide de très nombreuses petites mailles indépendantes et de seulement quelques grandes.

Ainsi, l'idée de la nouvelle stratégie (Nguyen et al., 2011a) s'appuie sur le couplage de deux algorithmes, chacun adapté à ces deux types de tailles de mailles indépendantes. D'une part, un algorithme de parcours de graphe va s'occuper, entre autres, de trouver les petites mailles indépendantes pour les regrouper en sous-circuit (il y en a plusieurs : figure 3 à droite). La détection est possible et très efficace du fait de la proximité des nœuds concernés. D'autre part, la méthode de détection générale est toujours utilisée mais n'est plus dévouée qu'à la détection des grandes mailles indépendantes. Si la complexité de ces mailles indépendantes est grande, leur nombre est très réduit. Ainsi, le temps de détection est limité. Ce traitement permet la construction de super-circuit (souvent un seul : figure 3 à droite).

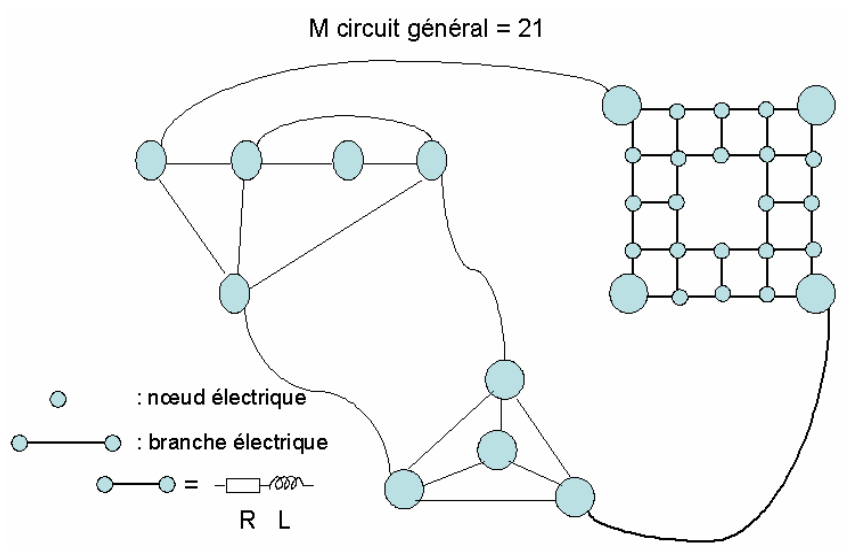

Figure 3. Principe simplifié de la méthode de détection des mailles indépendantes dans InCa3D actuel 
L'ensemble s'organise en trois étapes :

- Recherche des petites mailles indépendantes (MI) (constituées au maximum de 4 branches $)$. Le nombre de MI trouvées sur notre exemple est $18(3+12+3=18$ à comparer avec 21 au total - figure 3 ).

- Construction des sous-circuits à partir des informations des petites MI. On reste alors géométriquement dans la même zone et cela permet de regrouper les petites MI en sous-circuits (3 sous-circuits sur la figure 3). Toutes les mailles indépendantes dans des sous-circuits sont détectées par des algorithmes de graphe simples (Nguyen et al., 2011a).

- Les branches qui n'ont pas été traitées sont placées dans le ou les supercircuits et la méthode algébrique de détection générale est appliquée pour trouver des MI dans le ou les super-circuits. Sur l'exemple de la figure 4, on a donc $3+13+3=19$ mailles indépendantes. Pour arriver à 21 la méthode de détection générale est utilisée pour seulement 2 mailles indépendantes (figure 4).

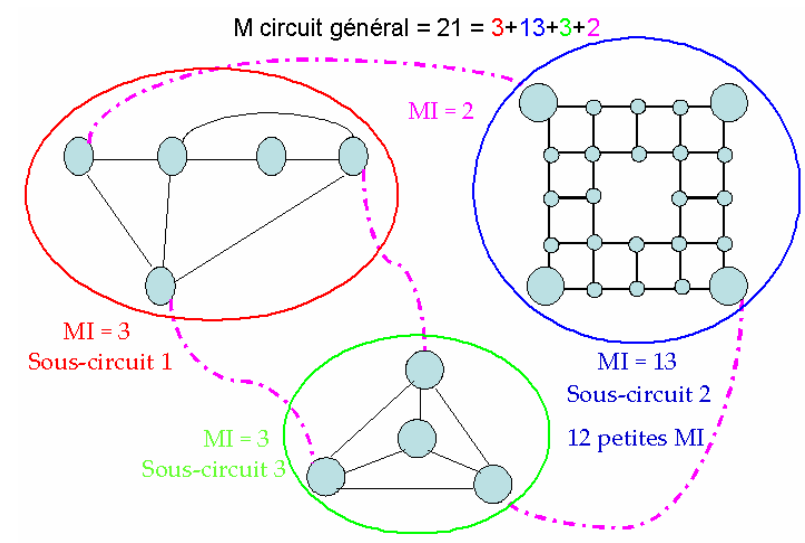

Figure 4. Principe simplifié de la méthode de détection des mailles indépendantes dans notre nouvelle approche

L'efficacité de cette nouvelle approche est illustrée dans la section 4.1. La pertinence de la méthode est confortée par le fait qu'elle donne des solutions rapides sur plusieurs exemples de circuits différents.

\subsubsection{Développement pour la résolution}

Principe de la FMM

Les algorithmes du type "Adaptative Multi-Level Fast Multipole Method" (AMLFMM) (Carrier et al., 1988) sont des algorithmes numériques qui sont très prometteurs pour les méthodes intégrales. L'approche classique consiste à exprimer l'ensemble des interactions d'un élément de courant avec tous les autres, quelle que 
soit la distance. Comme illustré figure 5, ce type d'approche aboutit à la construction d'une matrice d'interaction pleine, ce qui est fortement limitant dans le cas de grands systèmes à modéliser. L'approche proposée par un algorithme de type FMM, dont le principe simplifié est illustré sur la figure 5, permet de repousser considérablement ces limites.

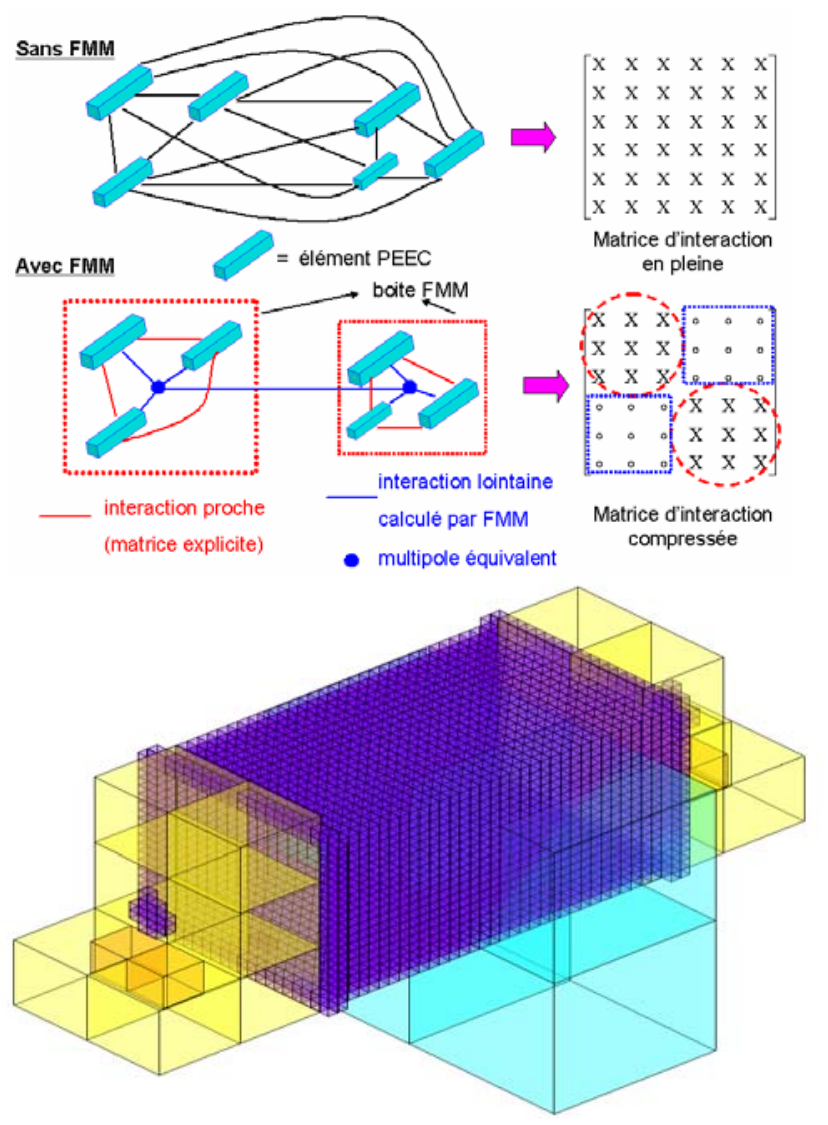

Figure 5. Principe de la FMM et partitionnement MLFMM au niveau 6 avec prise en compte de la taille des éléments

En restant dans une description de principe, la géométrie du problème est découpée en éléments parallélépipédiques de taille variable (figure 5). À partir de ce maillage volumique, deux types d'interactions sont maintenant distingués entre les éléments de courant. D'une part, lorsque les éléments de courant sont voisins (donc dans des volumes adjacents), on les considère en « interactions proches » et on les traite comme dans l'approche classique, c'est-à-dire en interaction complète 
(interaction rouge dans la figure 5). D'autre part, dès que les éléments considérés sont plus éloignés donc en «interactions lointaines» (dans des volumes non connexes), le couplage magnétique n'est plus calculé par la formule analytique exacte de la méthode PEEC mais est exprimé au travers du multipôle magnétique équivalent de la cellule. On ne considère alors plus qu'une seule interaction entre ces multipôles, comme illustré figure 5 en bas à gauche. L'interaction lointaine est calculée donc de la façon explicite sans stocker les termes dans la matrice d'interaction. La taille de chaque cellule multipolaire est pilotée par des critères d'approximation.

Ainsi, entre autres effets, ces algorithmes permettent de générer des matrices moins denses pour lesquelles il est en général nécessaire de stocker seulement entre 5 et $10 \%$ des termes. Le couplage de ces méthodes avec une technique de résolution itérative de systèmes linéaires de type GMres (Saad, 1993) permet d'avoir une complexité évoluant en $\mathrm{N} \log (\mathrm{N})$ grâce à l'accélération de calcul du produit matricevecteur par FMM, à comparer à une résolution par décomposition LU évoluant en $\mathrm{N}^{3}$.

Suite au succès obtenu avec l'application de cette technique pour le calcul des capacités parasites en PEEC (Ardon, 2010), une approche générale et robuste pour l'utilisation des FMM en modélisation inductive est mise en œuvre (figure 5). Il est à noter que son application à la modélisation d'un réseau de bord d'un avion constitue en soi une originalité puisque la présence d'éléments particuliers (conducteurs très longs) pose des problèmes de précision dans le calcul et de contrôle de l'erreur générée.

L'utilisation des FMM permet d'économiser la mémoire nécessaire au stockage de la matrice à résoudre mais la dimension de cette matrice reste encore très grande. Cela peut empêcher la convergence de la résolution du système (1) ; une technique de préconditionnement efficace est donc encore nécessaire pour améliorer, voire permettre, la convergence de la résolution. Une technique est présentée ci-après.

Préconditionnement avec GMres

Système à résoudre

Rappelons que le système d'équations à résoudre est :

$$
(\mathrm{G}+\mathrm{sC}) \cdot \mathrm{x}=\mathrm{Bu}
$$

où avec l'approche des mailles indépendantes $\mathbf{Z}_{\mathrm{m}}=\mathbf{G}+s . \mathbf{C}$.

Ainsi l'équation devient :

$$
\mathbf{Z}_{\mathbf{m}} \cdot \mathbf{I}_{\mathbf{m}}=\mathbf{V}_{\mathbf{m}}
$$

Avec :

$-\mathbf{Z}_{\mathbf{m}}$, la matrice d'impédance complexe issue de la méthode PEEC,

- Vm, le vecteur de sources de courants et de tensions,

- Im, le vecteur des inconnues. 
La solution du système (3) ne peut pas être trouvée par une méthode directe puisqu'elle suppose de connaître tous les termes de la matrice $\mathbf{Z}_{\mathbf{m}}$. Or, seuls les éléments PEEC en interaction proche sont stockés et regroupés dans la matrice creuse $\mathbf{Z}_{\mathbf{m} \_ \text {proche }}$. Ainsi, la matrice $\mathbf{Z}_{\mathbf{m}}$ peut être décomposée en deux parties $\mathbf{Z}_{\mathbf{m}}=$ $\mathbf{Z}_{\mathbf{m} \_ \text {proche }}+\mathbf{Z}_{\mathbf{m} \_ \text {lointaine }}$ où $\mathbf{Z}_{\mathbf{m} \_ \text {lointaine }}$ n'est pas stockée explicitement.

\section{Principe du GMres préconditionné}

Une première formulation de la résolution par GMres du système principal (3) est donnée sur la figure 6, à gauche. On la note GMres1 et elle s'appuie sur l'utilisation du préconditionneur noté P1. Le nouveau système préconditionné s'écrit alors :

$$
\mathrm{P1}^{-1} . \mathrm{Zm} . \mathrm{Im}=\mathrm{P1}^{-1} . \mathrm{Vm}
$$

Avec le solveur itératif GMres1, dans la $\mathrm{k}^{\mathrm{e}}$ itération, le système doit être résolu pour obtenir le résidu r1k qui s'écrit alors :

$$
\mathbf{P}_{1} \cdot \mathbf{r}_{1 \mathrm{pk}}=\mathbf{V}_{\mathrm{m}}-\mathbf{Z}_{\mathrm{m}} \cdot \mathbf{I}_{\mathrm{m}(\mathrm{k}-1)}=\mathbf{r}_{1 \mathrm{p}(\mathrm{k}-1)}
$$

dans laquelle $\mathbf{r}_{\mathbf{1 p ( k - 1 )}}$ est le résidu correspondant à l'itération précédente.

Choix du préconditionneur

La matrice Zm_proche est une bonne candidate pour préconditionner le système. En effet, d'une part, elle contient une information partielle du système, et, d'autre part, grâce à l'approche des mailles indépendantes, elle contient plus d'éléments situés sur sa diagonale. Donc nous avons choisi P1 $=$ Zm_proche comme une approximation de la matrice $\mathbf{Z m}$.

Ainsi, le $\mathrm{k}^{\mathrm{e}}$ résidu correspond à : $\mathbf{r 1 k}=\left(\mathrm{Zm} \_\right.$proche $)-1 . \mathbf{r 1}(\mathbf{k}-1)$

Ce calcul demande l'inversion du préconditionneur, ce qui n'est pas efficace du fait de sa taille. En évitant l'inversion, nous avons utilisé un autre GMres (solveur intérieur GMres2) qui demande aussi un autre préconditionneur P2. Il est choisi comme les blocs diagonaux de la matrice $\mathbf{Z m}$ _proche pour faciliter l'inversion de P2 (l'inversion de plusieurs matrices de petites tailles).

Le principe de ce processus d'imbrication des solveurs est illustré figure 6. Cette technique de double préconditionnement de type « inner-outer solveur GMrES » a été mise en place par Nguyen et al. (2011b). Basée sur les régimes de solveur imbriqué, elle est étudiée par de nombreux auteurs et a montré une bonne efficacité en électromagnétisme (Carpentieri et al., 2005 ; Saad, 1993). Notons que, pour être efficace, la résolution de GMres2 doit être plus rapide que GMres1. Ici, le deuxième processus de résolution peut conduire à un plus grand nombre d'itérations, mais le temps nécessaire doit rester plus petit. 


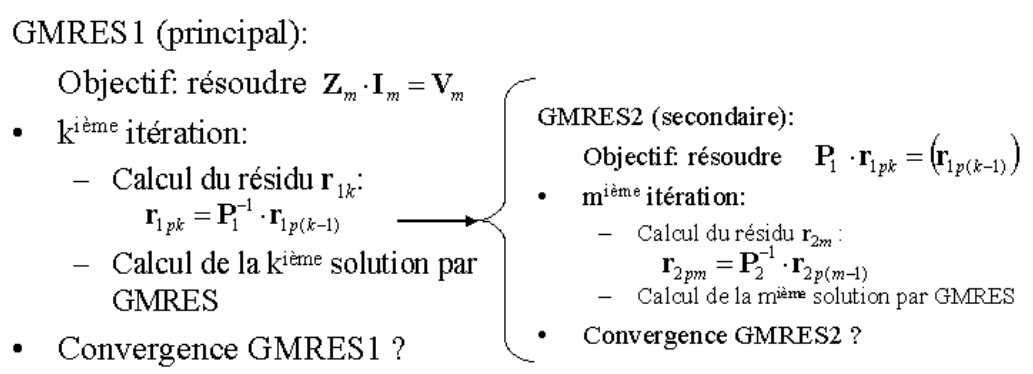

Figure 6. Principe de préconditionnement « inner-outer»

\section{Résultats sur le prototype « caisson »}

La géométrie du caisson présentée figure 1 est décrite dans le logiciel InCa3D. Dans un premier temps les performances des deux versions du solveur (version InCa3D actuelle et version développée) sont comparées sur une gamme de fréquences allant de $1 \mathrm{~Hz}$ jusqu'à $1 \mathrm{MHz}$. Les calculs de la distribution de courant sont ensuite présentés.

\subsection{Performances des résolutions}

Le temps nécessaire pour établir les équations de circuit par la nouvelle méthode est de $0,5 \mathrm{~s}$, et est 100 fois plus petit que l'ancienne méthode de détection des mailles indépendantes (tableau 1).

Tableau 1. Comparaison des performances entre versions de logiciel

\begin{tabular}{|l|c|c|c|}
\hline & Version actuelle & Version FMM & GAIN (fois) \\
\hline Temps de résolution (min) & 50 & 0,5 & 100 \\
\hline
\end{tabular}

En vue de la résolution totale, le tableau 2 se propose de comparer les performances de résolution entre la version actuelle du solveur et la nouvelle stratégie proposée basée sur les FMM. Il est important de noter que la première simulation ne peut aboutir que sur un serveur de calcul disposant de 32 Go de RAM et 8 processeurs. Par contre la version FMM fonctionne sur un PC standard Core 2 Duo avec 3 Go de mémoire vive. Le problème contient 11520 éléments de maillage (appelées subdivisions), 7442 nœuds mais seulement 5344 inconnues de type mailles indépendantes. Les développements réalisés doivent aussi conserver la pertinence physique des résultats. 
Tableau 2. Comparaison des performances entre versions de logiciel

\begin{tabular}{|l|c|c|c|}
\hline & Solveur actuel & Solveur FMM & Gain (fois) \\
\hline Mémoire utilisée (Mo) & 22000 & 900 & 24,4 \\
\hline Temps de résolution (min) & 532 & 159 & 3,3 \\
\hline
\end{tabular}

\subsection{Courants et densité du courant}

Après la résolution avec InCa3D sur une gamme de fréquences allant de $1 \mathrm{~Hz}$ jusqu'à $1 \mathrm{MHz}$, la densité de courant circulant dans le dispositif est tracée. D'après la figure 7 à gauche, il peut être remarqué qu'en basses fréquences la densité du courant circule uniformément sur le caisson. En s'approchant du point de sortie, la densité de courant croît pour retrouver la valeur qu'elle avait au point d'injection. En revanche, quand la fréquence augmente, le courant a tendance à circuler seulement sur les périphéries (figure 7 au milieu) ou sur les parties du caisson qui se trouvent en vis-à-vis des conducteurs (figure 7 à droite). Cela correspond à l'effet dit « de proximité » qui oblige le courant à se concentrer au droit des conducteurs. L'effet de peau sur le plan de masse métallique peut également être visualisé, car la densité du courant commence à circuler sur la périphérie de la plaque avec l'augmentation de la fréquence.
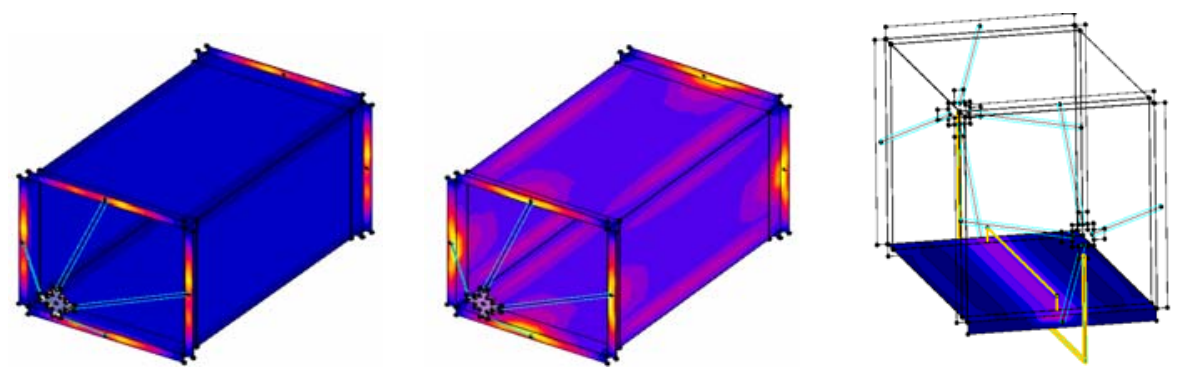

Figure 7. Simulations de la densité du courant, à $1 \mathrm{~Hz}$ (à gauche), à $1 \mathrm{MHz}$ (au milieu et à droite)

Des câbles, censés représenter les différents faisceaux d'une structure réelle, sont insérés à l'intérieur de la structure caisson. Par simulation, la répartition du courant dans les différents câbles et la proportion du courant circulant dans l'enveloppe sont ainsi modélisées. Cette répartition évolue avec la fréquence ainsi qu'avec la nature du matériau constituant le caisson (aluminium ou composite) comme détaillé dans Jazzar et al. (2011). 
Comme mentionné précédemment, des mesures sont effectuées et comparées aux simulations. Sur une gamme de fréquences de $1 \mathrm{~Hz}$ jusqu'à $1 \mathrm{MHZ}$, deux configurations sont par la suite détaillées (figures 8 et 9). Ceci permet de mettre en avant le degré de validité du modèle développé, du processus de modélisation et des avantages de l'outil utilisé.

Configuration 1. À l'intérieur du caisson, un câble d'une longueur de 1,05 m est connecté au milieu de la plaque 1 , et à une hauteur de $50 \mathrm{~mm}$ (figure 8 à gauche). Après injection d'un courant dans le caisson, le courant qui passe dans le câble est mesuré. Cette configuration est modélisée sur InCa3D et les deux résultats sont comparés sur la figure 8 , à droite.

Les résultats sont comparables sur toute la gamme de fréquence et se rapprochent de ceux trouvés dans la littérature: en basse fréquence le courant circule sur les parties les moins résistives, dans le cas étudié il s'agit du câble qui est à l'intérieur du caisson, et cela représente environ $50 \%$ du courant injecté. En revanche, en haute fréquence, le courant circule dans les parties les moins inductives constituées par les plaques en composite. Une très faible quantité de courant circule dans le câble.

On peut constater que les performances électriques du panneau en composite sont dégradées essentiellement en basse fréquence. Par contre, en haute fréquence il offre une protection électromagnétique équivalente à celle d'un panneau métallique.
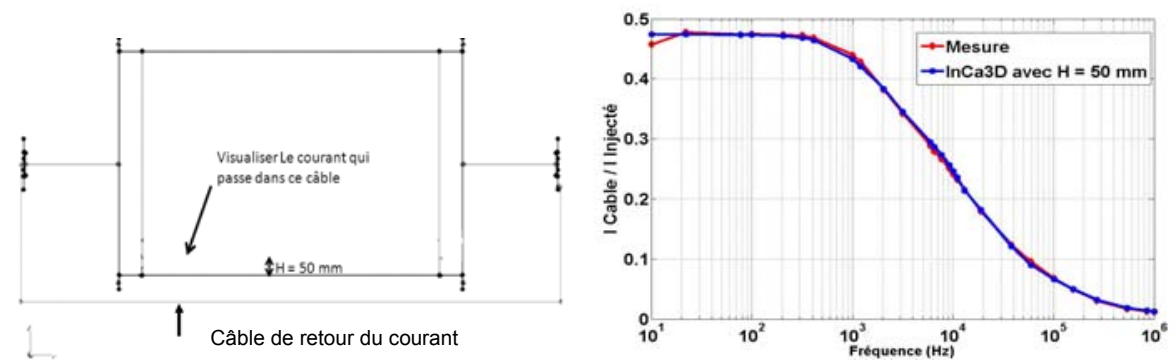

Figure 8. Comparaison des simulations et de la mesure avec $H=50 \mathrm{~mm}$

Configuration 2. Une plaque en aluminium connectée au milieu du caisson est rajoutée à la configuration précédente (figure 9 à gauche). Elle simule la présence d'un plancher. Sur la figure 9 à droite la proportion de courant dans le câble par rapport au courant injecté en simulation est comparée aux mesures.

La présence de cette plaque réduit la quantité de courant qui passe dans le câble de $50 \%$ à $15 \%$ en basses fréquences. Ceci est dû à la résistivité de la plaque en aluminium qui est plus petite que celle du câble. Ainsi au fur et à mesure que la fréquence augmente, la circulation du courant dans le dispositif va prendre en compte les phénomènes de couplage inductif. 

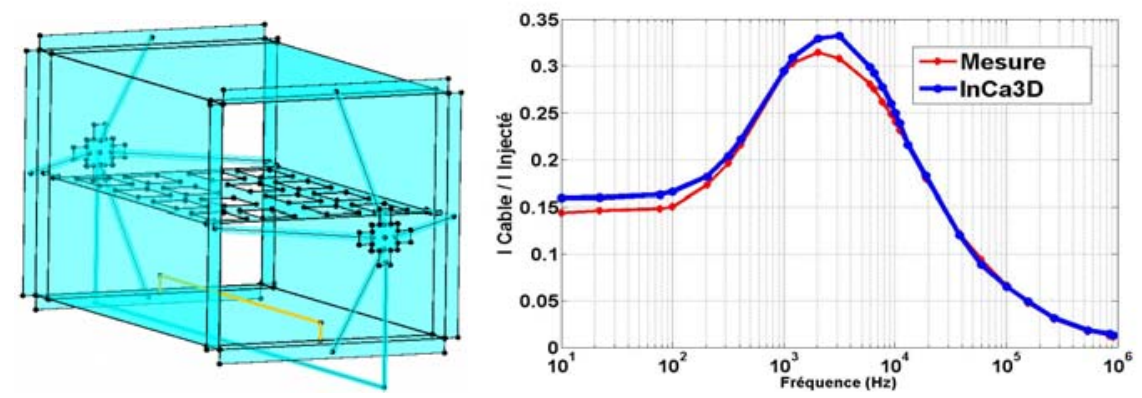

Figure 9. Comparaison des simulations et de la mesure

Ces résultats confirment la validité de la méthode PEEC pour ce type de calculs. Nous disposons donc d'une méthode efficace pour évaluer en fréquentiel les répartitions de courant dans les différentes parties d'un système pouvant être complexe.

Les expérimentations étant très délicates (voire coûteuses) sur ce genre de dispositifs, la simulation est un apport indéniable pour tester la pertinence de solutions envisagées. Ainsi ayant confiance dans le modèle généré, on peut donc s'autoriser à faire varier des paramètres afin, non seulement, de confirmer certaines tendances de comportement mais aussi les quantifier. Ceci est présenté dans la section suivante.

\section{3. Étude paramétrique}

Les systèmes électriques sont souvent reliés par des conducteurs très proches les uns des autres. Ce rapprochement des conducteurs augmente les interactions électromagnétiques entre eux. Cependant, plus les conducteurs sont proches les uns des autres plus l'effet de proximité s'ajoutant à l'effet de peau devient important. Ainsi, les performances varient fortement avec la fréquence et l'espacement entre conducteurs. Pour certaines configurations, l'augmentation de la résistance due à l'effet de proximité peut être de l'ordre de $100 \%$ supérieure à celle due à l'effet de peau seul.

Grâce à la simulation avec InCa3D ces différents phénomènes peuvent être modélisés.

L'effet de peau ainsi que l'effet de proximité peuvent être mis en évidence à la fois sur la répartition du courant mais aussi sur l'impédance équivalente des différents constituants du cas d'étude, chose délicate à faire en expérimentation.

Dans le cas présenté figure 8, l'augmentation de la hauteur entre les deux câbles (le premier dans lequel le courant est visualisé et l'autre étant le câble de retour) fait 
en sorte de diminuer les courants dans le premier câble, et du coup le comportement de l'impédance change sensiblement (surtout la partie inductive); ceci s'explique par le fait que la boucle créée par les deux câbles s'agrandit.

Grâce à la simulation, l'impact de la hauteur du câble sur la répartition du courant injecté dans le cas de la configuration 1 (figure 8) peut être évalué. La figure 10 montre le courant qui passe dans le câble pour deux valeurs du paramètre $\mathrm{H}(50 \mathrm{~mm}$ et $100 \mathrm{~mm})$.

Deux phénomènes interviennent. D'une part, en basses fréquences, quand $\mathrm{H}$ augmente, le courant dans le câble diminue de $47,5 \%$ jusqu'à $45 \%$. Ceci s'explique par l'augmentation de la résistance du câble avec H. D'autre part, au fur et la mesure que la fréquence augmente, on remarque que le courant qui passe dans le câble va continuer à diminuer. Ceci est dû au fait que l'inductance du câble simulé va augmenter à cause de l'augmentation de la surface entre le câble étudié et le câble de retour du courant.

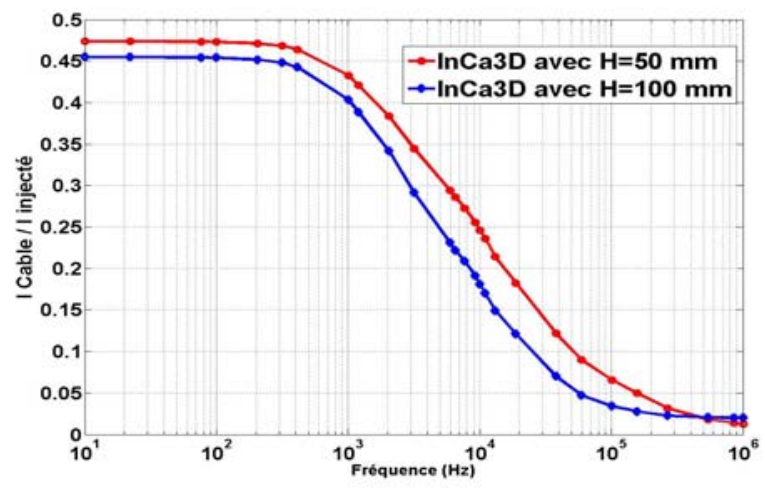

Figure 10. Comparaison en simulation avec $H=50 \mathrm{~mm}$ et $H=100 \mathrm{~mm}$

Les études paramétriques sont importantes pour les constructeurs de l'avion pour pouvoir évaluer et anticiper les phénomènes et l'évolution des contraintes qui en découle. Ceci dans le but de proposer les meilleures protections possibles adaptées au besoin et à l'environnement.

D'autres paramètres, qu'ils soient géométriques ou physiques, peuvent ainsi faire l'objet d'une analyse du même type.

Ainsi d'un point de vue physique, si l'on revient sur la nature du matériau du caisson et que ce dernier est tout en aluminium, la part du courant injecté circulant dans le câble en est fortement réduite comme le montre la figure 11. En effet, le câble est dans ce cas plus résistif et le courant choisissant le chemin de plus faible impédance circulera donc majoritairement dans les plaques du caisson montrant 
ainsi qu'en cas d'agression foudre, les structures internes plus impédantes que la carlingue sont en général épargnées.
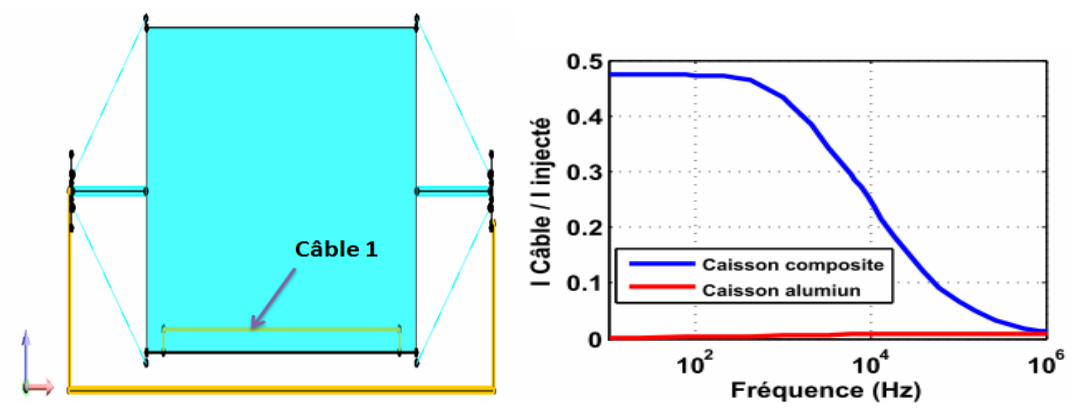

Figure 11. Comparaison en simulation avec un caisson en aluminium ou en composite

\subsection{Simulation temporelle}

Pour accéder à une information pertinente pour les industriels de l'aéronautique, un schéma équivalent du dispositif étudié sur une gamme de fréquences donnée doit être extrait. Ce circuit est ensuite implanté dans un logiciel de type circuit pour alors obtenir la réponse temporelle en l'alimentant avec une source qui permettra de décrire fidèlement la foudre.

Traditionnellement, les techniques de simulation dans le domaine temporel reposent sur la méthode FDTD. Mais, il est difficile de décrire finement la géométrie de l'aéronef ou du système (de l'avion entier aux parasites des câbles), car elle impose un pas temporel fin pour simuler des phénomènes de plusieurs centaines de microsecondes, ce qui accroît très rapidement les temps de calcul.

Dans cet article la plateforme logicielle (InCa3D + Portunus) est testée. La taille du schéma électrique équivalent est définie dans InCa3D à l'aide de sondes d'impédances qui vont permettre de donner les points d'entrée et sortie de la matrice impédance ainsi obtenue dont toutes les valeurs (partie réelle et imaginaire) sont fonction de la fréquence. Ces résultats sont exportés dans un fichier Portunus à partir d'InCa3D en créant un macro-bloc, présenté figure 12 à gauche. Formes et valeurs des courants sont obtenues après excitation du macro-bloc par un courant dont la forme d'onde est normalisée (figure 12 à droite) et est représentative du courant de foudre (Wave form A).

Dans le cas du caisson avec un seul câble à l'intérieur, deux éléments, caisson en composite, d'une part et câble en aluminium, d'autre part (donc deux sondes), sont identifiés et le schéma bloc correspondant est généré. Les éléments inductifs sur ce 
schéma électrique sont bien évidemment couplés par mutuelle inductance, graphiquement non représentée pour plus de clarté.

La forme d'onde $\mathrm{A}$ de la foudre possède deux régimes: lors des premières micro-secondes, le régime transitoire correspondant aux hautes fréquences s'installe pour se transformer après 10 micro-secondes, en régime permanent correspondant aux basses fréquences. En revanche, pour pouvoir simuler la forme d'onde du courant de foudre dans le macro bloc d'InCa3D, il faut avoir, sur la gamme de fréquences considérée, des résistances et des inductances de valeurs fixes, ce qui est vérifié dans ce cas. Des développements sont en cours pour synthétiser sous forme de schéma RLC, la répartition spectrale de l'inductance demandée lors du placement de la sonde.

D'après la figure 12 à droite, nous constatons qu'en hautes fréquences le courant circule davantage dans le matériau composite (moins inductif) et qu'en basses fréquences, le courant tend à ne circuler que dans l'aluminium (moins résistif).

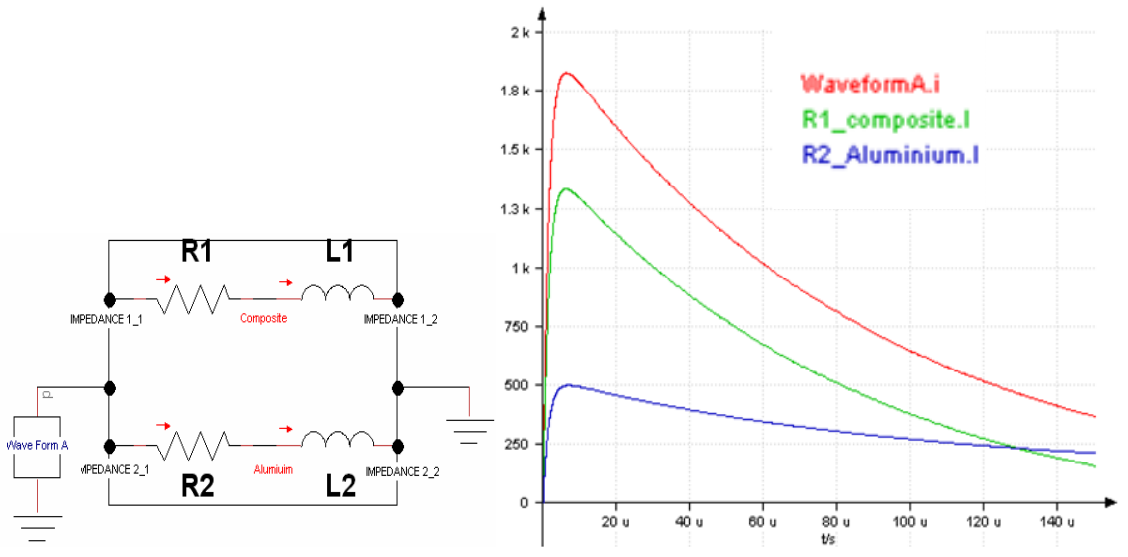

Figure 12. Injection du courant de foudre dans le macro-bloc d'InCa3D à gaucheRépartition du courant foudre dans le circuit équivalent à droite

\section{Conclusions et perspectives}

Grâce à l'application étudiée dans cet article, la méthode intégrale PEEC peut répondre à des besoins de simulations numériques sur des dispositifs complexes, comme les réseaux de bord des avions. Pour cela, cet article présente la méthodologie de modélisation, la nécessaire évolution des méthodes actuelles et les résultats obtenus grâce à ces améliorations qui visent à minimiser le temps de résolution et la taille du système. Le facteur 10 attendu est quasiment atteint. Dans un second temps, les nouvelles possibilités du solveur permettent de discuter de la 
répartition des courants et de l'évolution des impédances dans la structure composite en fonction de la fréquence.

Enfin, la réduction 3D vers $0 \mathrm{D}$ de la méthode PEEC permet d'exporter et de simuler temporellement les structures soumises aux sources d'excitation de foudre mais est dépendante de la fréquence. Afin de générer un schéma électrique équivalent indépendant de la fréquence plus aisé à utiliser en simulation temporelle, des développements doivent encore être entrepris. De nombreuses recherches visent à l'amélioration de ces méthodes (Tan et He, 2007) et plusieurs algorithmes sont disponibles dans la littérature. La solution envisagée pour notre étude se décompose en deux étapes. La première consiste, en partant de la représentation d'état, à ne garder que les états significatifs du système. Cela revient à calculer, autour de la fréquence retenue, un développement limité de l'impédance et à n'en garder que les moments d'ordres les plus faibles. En outre, la méthode utilisée permet de garantir la stabilité du système réduit (Tan et He, 2007). Dans un second temps, à partir de cette fonction de transfert réduite, une synthèse de circuit permet de reconstruire un schéma à constantes localisées, de taille compatible avec les capacités des simulateurs temporels. Cette deuxième étape est simplifiée du fait que les éléments réactifs du circuit synthétisé ne sont pas forcément réalistes (les inductances peuvent être de valeur négative par exemple, Tan et He, 2007).

\section{Remerciements}

Ce travail est réalisé dans le cadre d'un projet national d'envergure regroupant de nombreux partenaires et financeurs appelé PREFACE. Les auteurs tiennent à remercier ASTECH : Hispano-Suiza, AESE : Safran Engineering Service, PEGASE : Eurocopter.

\section{Bibliographie}

Ardon V. (2010). Méthodes numériques et outils logiciels pour la prise en compte des effets capacitifs dans la modélisation CEM de dispositifs d'électronique de puissance, thèse en Génie électrique, Université de Grenoble.

Carpentieri B., Duff I. S., Giraud L., Sylvand G. (2005). Combining fast multipole techniques and an approximate inverse preconditioner for large electromagnetism calculations, SIAM J. Sci. Comput., vol. 27, n 3, p. 774-792.

Carrier J., Greengard L., Rokhlin V. (1988). A fast adaptive multipole algorithm for particle simulations, SIAM J. Sci. Statist. Comput.

Clavel E., Meunier G., Bellon M., Lorang X. (2011). Caractérisation électromagnétique d'une boucle inductive d'aide au shuntage des trains, Actes de conférence Electrotechnique du Futur 2011, Belfort, France.

Gutiérrez G. G., Gil E. P., Gómez D. G., Gómez J. I. P. (2011). Finite-Difference TimeDomain Method applied to Lightning Simulation and Aircraft Certification Process, Proc. of the 10th Int. Symposium on EMC, York, UK. 
Jazzar A., Clavel E., Meunier G., Vincent B., Goleanu A., Vialardi E. (2011). Modeling and simulating the lightning phenomenon: aeronautic materials comparison in conducted and radiated modes. Actes de conférences IEEE-ISIE 2011, Gdansk, Poland.

Kamon M., Philips J. R. (1994). Preconditioning techniques for constrained vector potential integral equations, with application to 3-D magnetoquasistatic analysis of electronic packages, Actes de conférence on Iterative Methods 1994, Colorado.

Nguyen T.-S., Guichon J-M., Chadebec O., Meunier G., Vincent B. (2011a). An independent loops search algorithm for solving inductive PEEC large problems, Actes de conférences COMPUMAG 2011, Sydney, Australia.

Nguyen T.-S., Guichon J-M., Chadebec O., Meunier G., Le-Duc T. (2011b). Inner-outer preconditioning strategy for 3D inductance extraction coupling with Fast Multipole Method, Actes de conférences CEM 2011, Wroclaw, Poland.

Perrin E., Tristant F., Guiffaut C., Terrade F., Reineix A. (2012), A 3d model to compute lightning and hirf coupling effects on avionic equipment of an aircraft, ESA Workshop of Aerospace EMC, Venise, Italie.

Roudet J., Clavel E., Guichon J-M, Schanen J-L (2004). Modélisation PEEC des connexions dans les convertisseurs de puissance. Techniques de l'ingénieur, D307.

Ruehli A. E., Antonini G., Esch J., Ekman J., Mayo A., Orlandi A. (2003). Nonorthogonal PEEC Formulation for Time- and Frequency-Domain EM and Circuit Modeling, IEEE Transactions on EMC, vol. 45, n² 2, p. 167-176.

Saad Y. (1993). A flexible inner-outer preconditioned GMRES algorithm, SIAM J. Sci. Comput., 14, p. 461-469.

Tirkas P. A., Balanis C. A., Purchine M. P., Barber G. C. (1993), Finite-difference timedomain method for electromagnetic radiation, interference, and interaction with complex structures, IEEE Transactions on EMC, vo1. $35, \mathrm{n}^{\circ} 2$.

Tan S. X.-D., He L. (2007). Advanced Model Order Reduction Techniques in VLSI Design. Cambridge University Press. 